D. Schadendorf ${ }^{1} \cdot$ G. Schuler ${ }^{2}$ A. Paschen ${ }^{1} \cdot$ C. Scheibenbogen ${ }^{3}$

${ }^{1}$ Klinische Kooperationseinheit für Dermatoonkologie (DKFZ), Klinik für Dermatologie, Venerologie und Allergologie, Universitätsklinikum Mannheim

${ }^{2}$ Universitätshautklinik Erlangen

${ }^{3}$ Klinik für Hämatologie und Onkologie, FU Berlin

\title{
Experimentelle Therapie des Melanoms
}

\section{Tumorimmunologische Grundlagen}

pie oder die Bestrahlung sind nur bei wenigen Tumorentitäten wie z. B. kindlichen Leukämien gut wirksam. Das maligne Melanom ist in seinem fortgeschrittenen Krankheitsstadium durch eine hohe Mortalität gekennzeichnet. Dies ist u. a. durch die hohe Resistenz gegenüber konventionellen Therapiestrategien einschließlich Zytostatika bedingt. Daher ist es beim Melanom besonders wichtig, diesen Tumor früh zu diagnostizieren und chirurgisch zu eliminieren sowie neue Therapieansätze zu entwickeln.
Ziel ist es, mit der destruktiven Kraft des Immunsystems Tumorzellen selektiv zu vernichten. Klinische Beobachtungen bei Melanompatienten deuten auf eine besonders heftige, gegen den Tumor gerichtete, immunologische Abwehrreaktion hin. Dazu zählen das Auftreten von spontanen Melanomenregressionen, das lange Intervall zwischen dem Auftreten des Primärtumors und nachfolgender Melanommetastasierung, das in Einzelfällen mehr als 10 Jahre betragen kann, und das Auftreten von begleitender Vitiligo.

Basierend auf diesen Beobachtungen wurde das Konzept, das maligne Melanom in seinem Krankheitsverlauf durch immunologische Therapieansätze zu beeinflussen, entwickelt. So waren die letzten Jahrzehnte durch eine Vielzahl von Bemühungen geprägt, den klinischen Verlauf des Melanoms durch eine unspezifische Stimulation des Immunsystems insbesondere durch BCG, Levamisol, Corynebacterium parvum, Interferone und viele andere Substanzen zu beeinflussen [15]. Diese Bemühungen hatten jedoch keinen signifikanten Effekt auf die Rezidiventwicklung und die Gesamtüberlebenszeit.

Das Melanom stellt trotz seiner Aggressivität ein bevorzugtes Setting für die Exploration antigenspezifischer Immuntherapien dar. Gründe sind u. a. die große Zahl identifizierter Tumorantigene, der Nachweis von (u. U. allerdings funktionell inkompetenten) tumorspezifischen T-Zellen auch ohne Vakzination sowie die Rückbildung von Metastasen durch IL-2-in-vitro-expandierte und dann applizierte TIL (tumor infiltrating lymphocytes) [15, 20, 25, 27]. Die zytotoxische Effektorfunktionen dieser TIL oder aus dem Blut gewonnener zytotoxischer T-Lymphozyten (CTL) erlaubten nun, diese als Werkzeuge zu benutzen, um die erkannten Antigene zu identifizieren. Die Spezifität der Erkennung wird auf Seiten des T-Lymphozyten durch seinen T-Zell-Rezeptor gewährleistet und auf Seiten der Tumorzelle durch die einzelnen HLA-Moleküle an der Zelloberfläche, die jeweils verschiedene Peptide "geladen" haben. Nur wenn ein HLAPeptid-Komplex auf einen T-Lymphozyten mit dem passenden T-Zell-Rezeptor trifft (Schlüssel-Schloss-Prinzip), kommt es zur Erkennung und zur Zytolyse. Die Verfügbarkeit spezieller tumorspezifischer T-Zell-Klone in vitro, die aus Melanommetastasen oder aus dem Blut von Melanompatienten gewonnen wurden, führte in den letzten Jahren zur Identifizierung verschiedener Tumorantigene d. h. der Moleküle von denen Peptide durch die HLA-Moleküle an der Oberflä-

Prof.Dr. Dirk Schadendorf Klinische Kooperationseinheit für Dermatoonkologie (DKFZ, D0900), Klinik für Dermatologie, Venerologie und Allergologie, Universitätsklinikum Mannheim, Theodor-Kutzer Ufer 1, 68135 Mannheim, E-Mail:d.schadendorf@dkfz.de 
che präsentiert werden und die für eine Erkennung und Zytolyse von Melanomzellen verantwortlich sind. Beim Melanom gelang es so, in den letzten 10 Jahren mehr als 25 melanomassoziierte Antigene zu identifizieren (Übersicht bei Sun et al. [25]), die nachfolgend in 2 große Kategorien $\mathrm{zu}$ unterteilen waren:

1. die Gruppe der so genannten Differenzierungsantigene, die auch in normalen Melanozyten zu finden sind und mit der Pigmentsynthese assoziiert sind, wie die Tyrosinase, gp10o/Pmel 17, gp75/TRP-1 sowie das MelanA/MART-1,

2. eine Gruppe von Antigenen, die sowohl in Melanomen als auch in epithelialen Karzinomen, jedoch nicht in normalen Geweben mit Ausnahme von Hodengewebe exprimiert wird. Hierzu gehören die MAGEFamilie und verwandte Moleküle.

Für die meisten der oben erwähnten Antigene konnten bislang 1 oder 2 Peptide mit jeweils 9-10 Aminosäuren identifiziert werden, die durch spezifische, vornehmlich CD8+-T-Zellen in Abhängigkeit vom HLA-Molekül erkannt werden. CD8-positive T-Lymphozyten erkennen physiologischerweise kurze, 8-10 Aminosäuren lange Peptide, die im Rahmen der intrazellulären Proteolyse in jeder Zelle von jedem Protein entstehen und dann in Assoziation mit einem HLAMolekül - einem Fahrstuhl ähnlich - an die Zelloberfläche gelangen.

Da bereits eine Reihe derartiger Peptide beim Melanom identifiziert wurden, ist ihre Verwendung in Vakzinationsprotokollen beim malignen Melanom eine äußerst interessante Therapieoption, die derzeit weltweit an verschiedenen Orten evaluiert wird. Allen Protokollen gemeinsam ist das Ziel, eine zytotoxische T-Zell-Antwort gegen den Tumor zu erzeugen. Verschiedene Wege können hierzu beschritten werden:

1. Peptidvakzination in Kombination mit Adjuvanzien,

2. Verwendung dendritischer Zellen (DZ) als Vehikel und Adjuvans,

3. Einbringen der Tumorantigene mittels bakterieller oder viraler Vehikel, 4. Immunisierung mit der genetischen Information, die für das Tumorantigen kodiert („genetische Immunisierung“, „nackte DNA“).
In allen Fällen ist es notwendig, dass TZellen („naive oder memory T-Zellen“) in der Zirkulation und im Gewebe aktiviert werden, um immunologisch effektiv zu sein, d. h. T-Lymphozyten müssen lernen, das „richtige“ Peptid zu erkennen, um sich als Antwort auf die Antigenerkennung in großer Zahl zu vermehren. Naive T-Zellen sind darauf angewiesen, in einer "helfenden“ Umgebung mit dem zu erkennenden Antigen in Kontakt zu kommen, um später eine Zytotoxizität zu entwickeln. Diese Helferfunktion wird in der Regel durch Adhäsionsmoleküle auf speziellen, so genannten professionellen, Antigen-präsentierenden Zellen oder durch Zytokine erfüllt. Antigen-präsentierende Zellen sind in vielen Geweben unter verschiedenen Namen zu finden, u. a. als Mikrogliazellen im Hirn, Kupffer-Sternzellen in der Leber oder als Langerhans-Zelle in der Haut. Gemeinsam ist ihnen die Morphologie mit vielen Ausläufern, so genannten Dendriten, von denen auch der Name, ,dendritische Zellen“ stammt, sowie ihre Fähigkeit, kleinste Mengen von Fremdstoffen aufzunehmen und dem Immunsystem im Lymphknoten zu präsentieren (Übersicht bei Bell et al. [1]). Die Tatsache, dass sich dendritische Zellen in verhältnismäßig großer Zahl und reproduzierbar aus dem peripheren Blut herstellen lassen, haben sie zu einem hervorragenden Vakzinvehikel gemacht, dessen Funktionalität und Effektivität derzeit in zahlreichen Phase-I/II-Studien untersucht wird.

Eine andere Form der Krebsimpfung beruht auf der Applikation von nackter DNA. Dazu werden molekularbiologischen Konstrukte herstellt, die die genetische Information für die zuvor genannten antigenen Peptide beinhalten. Diese molekularbiologischen Konstrukte werden als nackte DNA i. m. appliziert, sodass die Peptide über längere Zeit produziert werden und $\mathrm{zu}$ einer starker T-Zell-Antwort und auch zur Antikörperproduktion führen. Unabhängig von den Möglichkeiten, die aus der Anwendung nackter DNA resultieren, werden in der Grundlagenforschung weitere Therapieansätze für die Klinik vorbereitet. Dazu zählt auch die Anwendung von Pockenviren, die früher für Pockenschutzimpfungen eingesetzt wurden, oder die Anwendung von Bakterien als Vehikel, um Tumorantigene in den Organismus des Patienten zu schleusen.

\section{Vakzination mit Peptiden}

Die Charakterisierung HLA-Klasse-Ibindender Peptidsequenzen aus melanomassozierten Proteinen als Zielstrukturen für zytotoxische T-Lymphozyten (CTL) war die Grundlage für die Entwicklung antigenspezifischer Tumorimpfstoffe. HLA-Klasse-I-bindende Peptide bestehen aus 9-10 Aminosäuren und lassen sich relativ einfach synthetisch herstellen. Peptide können ohne Antigenprozessierung direkt an HLAKlasse-I-Moleküle auf der Zelloberfläche binden. Ein Nachteil von Peptidimpfstoffen ist die HLA-Restriktion der Peptide, d. h. in Abhängigkeit vom HLATyp des Patienten können nur selektiv

\begin{tabular}{|c|c|c|c|c|c|}
\hline Antigen & Adjuvans & Patienten & $\begin{array}{l}\text { Klinisches } \\
\text { Ansprechen }\end{array}$ & $\begin{array}{l}\text { T-Zell- } \\
\text { Antwort }\end{array}$ & Referenz \\
\hline MAGE-3 & - & 39 & $\begin{array}{l}7 \text { CR/PR (25 } \\
\text { auswertbar) }\end{array}$ & $0 / 4(\mathrm{Cr})$ & 12 \\
\hline Tyrosinase/MART/Gp100 & GM-CSF & 3 & $3 \mathrm{CR} / \mathrm{PR}$ & $3 / 3(\mathrm{Cr})$ & 6 \\
\hline MART-1 & IFA & 18 & Nein & $12 / 18(\mathrm{Cr})$ & 2 \\
\hline Gp100 & $\begin{array}{l}\text { IFA } \\
\text { IFA/IL-2 i.v. }\end{array}$ & $\begin{array}{l}11 \\
19\end{array}$ & $\begin{array}{l}3 \mathrm{MxR} \\
8 \mathrm{CR} / \mathrm{PR}, \\
3 \mathrm{MxR}, 3 \mathrm{SD}\end{array}$ & $\begin{array}{l}10 / 11 \\
3 / 19 \text { (ELISA) }\end{array}$ & 17 \\
\hline Tyrosinase & GM-CSF & 16 & $1 M \times R, 2 S D$ & 2/13 (ELISPOT) & 20 \\
\hline
\end{tabular}

IFA inkomplettes Freund-Adjuvans; Cr Chromium-release-Assay 
Tabelle 2

Abgeschlossener Peptidvakzinestudien bei Melanompatienten in der adjuvanten Situation

\begin{tabular}{llclc} 
Antigen & Adjuvans & Patienten & T-Zell-Antwort & Referenz \\
\hline MAGE-3 & IFA, PADRE & 18 & $5 / 14$ (Cr) & {$[30]$} \\
MART-1 & IFA & 25 & $12 / 20$ (ELISPOT) & {$[28]$} \\
Tyrosinase & QS21 & 9 & $2 / 9$ (ELISPOT)) & {$[11]$} \\
Tyrosinase & GM-CSF & 11 & $7 / 11$ (ELISPOT) & {$[8]$} \\
Tyrosinase/Gp100 & $+/-$ IL-12 & 24 & $20 / 24$ (ELISA) & {$[31]$}
\end{tabular}

Peptide für den individuellen Patienten ausgewählt werden. Zudem ist die Charakterisierung einzelner Peptidepitope sehr aufwändig, sodass bislang v. a. Peptidepitope für HLA-A2.1, das bei nahezu 50\% der kaukasischen Bevölkerung vorkommt, bekannt sind (Übersicht bei Sun et al. [25]).

\section{Klinische Studien}

Erste Phase-I-Studien mit dem Ziel, Immunogenität und Toxizität einer Peptidvakzination zu überprüfen, wurden bei Patienten mit metastasiertem Melanom durchgeführt (Tabelle 1). Die Ergebnisse dieser ersten Studien sind viel versprechend, denn bei einzelnen Patienten wurden auch Tumorremissionen beobachtet. So konnte in einer Multizenterstudie durch Vakzination mit dem MAGE-3.A1-Peptid bei 7 von 25 Melanompatienten mit vorwiegend Hautmetastasen eine partielle oder komplette Remission einzelner Metastasen erreicht werden [13]. Auch Jäger et al. [7] berichteten von 3 Patienten, bei denen es nach Vakzination mit einem Cocktail HLAA2.1 bindender Peptidepitope der Antigene Tyrosinase, MART-1 und gp1oo zusammen mit dem Adjuvans GM-CSF zu einer teilweisen oder vollständigen Rückbildung der Metastasen kam.

Das Monitoring der T-Zell-Antwort mit in vitro expandierten T-Zell-Klonen, die im Chromium-release-Assay oder ELISA auf Antigenspezifität getestet wurden, erwies sich nicht als hilfreicher Surrogatmarker für die klinische Wirksamkeit. In der Studie von Marchand et al. [13] mit dem MAGE-3.A1Peptid gelang bei keinem Patienten der Nachweis einer T-Zell-Antwort. Umgekehrt konnte in mehreren Studien, die am NCI mit Peptiden der Melanomantigene MART-1 oder gp1oo in Kombinati- on mit inkomplettem Freund-Adjuvans (IFA) durchgeführt wurden, die Induktion einer T-Zell-Antwort bei den meisten Patienten nachgewiesen werden, obwohl nur wenige Patienten ein klinisches Ansprechen zeigten $[2,18]$. Auch die Vakzination mit Tyrosinasepeptiden in Kombination mit GM-CSF zeigte in einer Phase-II-Studie bei Patienten mit metastasiertem Melanom nur eine geringe klinische Wirksamkeit [21]. Eine Induktion spezifischer T-Zellen, die hier mittels ELISPOT-Assay direkt aus dem peripherem Blut bestimmt wurde, ließ sich jedoch auch nur bei 2 Patienten mit einem Mixed response und einer Tumorstabilisierung nachweisen. Inwieweit das Monitoring der T-Zell-Antwort direkt aus unstimulierten Lymphozyten mittels ELISPOT-Assay besser mit dem klinischen Ansprechen korreliert, muss im Rahmen von weiteren Studien geklärt werden. Die Ergebnisse der bislang publizierten Studien bei Patienten mit metastasiertem Melanom sind in Tabelle 1 aufgeführt.

Diese Studien sind die Grundlage für die Prüfung von Peptidvakzinen in der adjuvanten Situation $[8,11,28,30]$ (Tabelle 2). Die Wirksamkeit jeder Vakzination ist bei Patienten mit metastasiertem Melanom aufgrund der großen Tumorzellzahl und durch eine tumorbedingte Suppression der T-Zell-Antwort aller Wahrscheinlichkeit nach a priori begrenzt. In einer adjuvanten Phase-IStudie konnte gezeigt werden, dass Patienten, die eine T-Zell-Antwort gegen das MART-1-Peptid aufbauten, länger rezidivfrei blieben [28]. Im Rahmen einer groß angelegten 4-armigen randomisierten Studie (Peptide/GM-CSF vs. Peptide vs. GM-CSF vs. Plazebo) wird derzeit die Vakzination in der adjuvanten Situation innerhalb der amerikanischen Eastern Cooperative Oncology Group
(ECOG) geprüft. Eine ähnliche Studie ist in der EORTC Melanomgruppe in Vorbereitung.

\section{Bedeutung von immunologischen Adjuvanzien}

Welche Bedeutung immunologische Adjuvanzien bei der Peptidvakzination haben, wurde bislang kaum systematisch untersucht. Unter der Annahme, dass Tumorantigene als körpereigene Proteine relativ schwache Immunogene sind, wurden in den meisten Studien Adjuvanzien eingesetzt, die entweder, wie z. B. GM-CSF, die Antigenpräsentation stimulieren oder die Generierung von zytotoxischen T-Zellen unterstützen sollen. Jäger et al. [7] berichteten von einer höheren Immunogenität und Induktion von Remissionen durch die Zugabe von GM-CSF bei 3 Patienten, die sequenziell zunächst mit Peptiden allein und dann in Kombination mit GM-CSF vakziniert wurden. Die oben zitierte, randomisierte Studie der ECOG wird auch die Wirksamkeit von GM-CSF als Adjuvans klären. Rosenberg et al. [18] haben in einer nicht randomisierten Studie Patienten mit einem gproo-Peptid ohne und mit Hochdosis-IL-2 behandelt und nur mit IL-2 bei 42\% der Patienten objektive Tumorremissionen gesehen. Da IL-2 allein jedoch bei Patienten mit metastasiertem Melanom Remissionen induziert, ist die Wirksamkeit von IL-2 als Adjuvans durch diese Studie nicht eindeutig belegt. Auf dem ASCO-Kongress 2000 wurde eine laufende randomisierte Studie von Weber et al. [28] vorgestellt, in der Hochrisikopatienten adjuvant Peptide mit und ohne IL-12 i. d. erhalten. Die Auswertung der ersten 24 Patienten zeigte eine Steigerung der Immunogenität durch IL-12-Zugabe.

\section{Toxizität der Peptidvakzination}

Die häufig beobachteten Nebenwirkungen einer Peptidvakzination in Form von Entzündungsreaktionen an der Einstichstelle und „grippeähnlichen Symptomen" sind v. a. auf die begleitend eingesetzten Adjuvanzien zurückzuführen. Vitiligoähnliche Hautveränderungen wurden bei einzelnen Patienten nach Vakzination gegen Differenzierungsantigene beschrieben. Klinisch bedeutsame Nebenwirkungen wurden bislang nicht berichtet. 


\section{Weiterentwicklung von Vakzineansätzen}

Voraussetzung für eine konsequente klinische Entwicklung und Optimierung von Vakzinationsstrategien mit Tumorantigenen sind Messsysteme, mit denen die Induktion einer T-Zell-Antwort quantitativ und qualitativ bestimmt werden kann. Dies ist durch neue sensitive Testsysteme möglich, wie den ELISPOT-Assay, die eine direkte Analyse der T-Zell-Antwort ohne vorherige In-vitroStimulation erlauben $[8,11,21,26]$. Daher sollte die Vakzination mit Peptiden systematisch mit Hilfe sensitiver T-ZellAssays weiterentwickelt werden. Im Rahmen von Phase-I/II-Studien sollten zunächst Impfstrategien etabliert werden, die reproduzierbar eine T-Zell-Antwort generieren, bevor ein weiterer therapeutischer Einsatz im Rahmen von Phase-IIb- oder -III-Studien propagiert werden kann. Innerhalb der EORTCMelanomgruppe wird in Phase-I-Studien zurzeit die Immunogenität von Peptidvakzinen in Kombination mit unterschiedlichen Adjuvanzien geprüft. Eine randomisierte Phase-III-Studie in der adjuvanten Situation bei Hochrisikopatienten ist in Vorbereitung.

\section{Vakzination mit dendritischen Zellen}

Die Vakzination mit dendritischen Zellen (DZ), d.h. die Injektion antigenbeladener DZ in Patienten, ist eine besonders viel versprechende Strategie, um die Induktion tumorspezifischer T-Zellen und damit das wesentlichste Ziel der antigenspezifischen Immuntherapie $\mathrm{zu}$ erreichen. Von besonderer Bedeutung sind die CD8+-zytotoxischen T-Zellen (CTL), welche Tumorantigene bzw. daraus abgeleitete, 8-9 Aminosäuren lange Peptide in Assoziation mit MHC-KlasseI-Molekülen erkennen und so Tumorzellen lysieren können. Tierexperimente zeigen, dass für eine effektive und andauernde CTL-Antwort und Tumorkontrolle zusätzlich tumorspezifische CD4+-Helfer T-Zellen wichtig sind. Solche Zellen, welche aus Tumorantigenen hergeleitete, etwa 12-20 Aminosäuren lange Peptide in Assoziation mit MHCKlasse-II-Molekülen erkennen, wurden jüngst auch für Melanomantigene identifiziert. Diese CD4+-T-Zellen können Zytokine freisetzen, aber z. T. auch Klas-
se-II-positive Tumorzellen lysieren (Übersicht bei Wang [27]).

In die bislang nebenwirkungsarme DZ-Vakzination werden derzeit große Erwartungen gesetzt, obgleich die Umsetzung dieser Strategie noch ganz am Anfang steht. Der Optimismus gründete sich vorerst auf konzeptionelle Überlegungen, wurde dann aber durch die positiven Ergebnisse zahlreicher tierexperimenteller und auch erster klinischer Studien gestützt.

Das von Ralph M.Steinman und seinen Schülern über Jahre gegen breiten Widerstand entwickelte Konzept [6], dass DZ als „Adjuvans der Natur“ für die Einleitung von Immmunantworten essenziell sind, ist heute ebenso akzeptiert wie die Vorstellung der Wirkungsweise dieser Zellen. Unreife DZ in der Peripherie können perfekt Antigene aufnehmen und prozessieren, aber nur schlecht ruhende T-Zellen stimulieren. Erst wenn sie auf geeignete Stimuli hin (z. B. LPS oder inflammatorische Zytokine) reifen, exprimieren sie eine große Zahl immunogener MHC-Peptid-Komplexe sowie spezielle Chemokinrezeptoren und Adhäsions- bzw. Kostimulatormoleküle, die sie dann in die lymphoiden Organe wandern und dort antigenspezifische T-Zellen sensibilisieren lassen. Der Ansatz, Immunität dadurch zu induzieren, dass DZ ex vivo mit Antigen beladen und dann die beladenen DZ injiziert werden, ergibt sich als logische Konsequenz aus dieser biologischen Abfolge (Übersicht bei Schuler u. Steinman [22]). Besonders attraktiv erscheint der Ansatz auch deshalb, weil einerseits andere Vakzinationsstrategien ihre Wirkung - nicht wirklich überraschend - über die in vivo vorhandenen DZ entfalten (müssen) [22], diese präexistenten $\mathrm{DZ}$ aber andererseits gerade bei Tumorpatienten alteriert und u. U. sogar tolerogen sein können.

Die vorausgesagte potente immunogene Effektivität der DZ-Vakzination wurde erstmals 1990 von K. Inaba et al. [6] unter Verwendung von aus der Milz von Mäusen direkt isolierten DZ experimentell eindrucksvoll bestätigt. Erst die Anfang 1992 erstmals gezeigte Möglichkeit der In-vitro-Generation definierter und vollwertiger DZ unter Verwendung von GM-CSF erlaubte in der Folge die Durchführung breiter angelegter tierexperimenteller Studien insbesondere auf dem Gebiete der Tumorimmunologie (Übersicht bei Schuler u. Steinman
[22]). Die mit in vitro aus Knochenmarkvorläufern generierten, vorwiegend „reifen“ DZ-Populationen erfolgten Vakzinationen erwiesen sich im direkten Vergleich (soweit durchgeführt) mit anderen Vakzinationsstrategien überlegen und therapeutisch effektiv.

Auch beim Menschen wurden, wie bei der Maus, direkt aus dem Blut isolierte, präformierte DZ für die erste DZVakzination beim Menschen eingesetzt. Schuler u. Steinman [22] vakzinierten Patienten mit B-Zell-Lymphom mit dem Ig-Idiotypen ihres Tumors und berichteten 1996 über klinische Erfolge, die allerdings bislang durch keine größere Nachfolgestudie bestätigt wurden. Beim Menschen können DZ in vitro entweder aus den seltenen, proliferierenden CD34+-Vorläufern oder den häufigen, kaum proliferierenden CD14+-monozytären Präkursoren gezüchtet werden (Übersicht bei Schuler u. Steinman [22]). Die CD34+-basierte Methode ist nur bei der Verwendung von Nabelschnurblut simpel, beim Erwachsenen ist die Methode hingegen weit weniger attraktiv, da sie eine Vorbehandlung des Patienten mit z. B. G-CSF zur Erhöhung der Frequenz der $\mathrm{zu}$ isolierenden $\mathrm{CD}_{34+-Z e l-}$ len erfordert, die In-vitro-Generation der DZ langwierig ist und zu keinen reinen DZ-Populationen führt. Es überrascht daher nicht, dass bislang nur eine Pilotstudie (bei Stadium-IV-Melanompatienten) vorliegt [12].

Diese Methode wird ebenso wie die direkte Isolierung der komplexen Populationen präformierter DZ aus dem Blut in Zukunft wohl nur eine Rolle spielen, wenn sich in Vakzinationsstudien eklatante Vorteile beim direkten Vergleich mit aus CD14+-Monozyten generierten DZ (Mo-DZ) belegen lassen. Monozyten entwickeln sich unter dem Einfluss von GM-CSF und IL-4 (oder GM-CSF und IL-13) in „unreife Mo-DZ“, die durch diverse Reifungsstimuli (u. a. durch monozytenkonditioniertes Medium bzw. dessen Inhaltsstoffe IL-1 und IL-6 und TNF- und $\mathrm{PGE}_{2}$ ) dann in eine sehr homogene Population „reifer Mo-DZ“ übergeführt werden können. Seit der Erstbeschreibung dieses Ansatzes 1994 wurde die Methode systematisch weiterentwickelt, um die problemlose klinische Anwendung zu ermöglichen (z. B. durch Vermeidung von FCS bzw. fetalem Kälberserum) [22, 26] bzw. zu erleichtern (durch Herstellung gebrauchsferti- 
ger, mit Antigen beladener kryopräservierter DZ-Aliquots). Für die Mo-DZ liegen die meisten Erfahrungen hinsichtlich der Anwendung am Menschen vor. Dhodapkar et al. [3] zeigten, dass eine einzige subkutane Injektion von 2-4 Mio. antigenbeladener „reifer Mo-

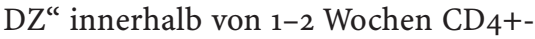
und CD8+-Effektoren im Blut gesunder Freiwilliger induzierte. Einige Monate nach dieser Impfung waren diese im Blut verschwunden, aber eine Auffrischungsimpfung mit „reifen Mo-DZ“ bewirkte eine quantitativ noch stärkere Reaktion, die sich auch qualitativ durch eine höhere Affinität der T-Zellen auszeichnete. Diese bislang bei keinem anderen Adjuvans berichteten Effekte stellen den derzeit wohl überzeugendsten Beleg für die Effektivität der DZ-Vakzinations-Strategie dar.

Pioniere auf dem Gebiet der Vakzination von Melanompatienten waren Nestle et al. [14], welche unter direkt intranodal erfolgenden Vakzinationen (jeweils nur 1 Mio. DZ, 4 Injektionen in wöchentlichen, dann 1 nach 2 Wochen, dann weitere in 4-monatlichen Abständen) erstaunliche klinische Effekte beobachten konnten (5/16 Patienten zeigten objektivierbares Ansprechen inklusive 2 CR nach 6-10 Wochen). Die DZ waren in FCS-haltigem Medium aus Frischblut gezüchtete "unreife Mo-DZ", welche jeweils gleichzeitig mit mehreren Melanompeptiden oder autologen $\mathrm{Tu}$ morzelllysaten (2 Patienten) sowie KLH (als unspezifischem Helferprotein) beladen worden waren. Die Effekte sind aus heutiger Sicht erstaunlich, da Jonuleit et al. (Manuskript zur Publikation eingereicht) jüngst bei einem direkten Vergleich intranodal injizierter Mo-DZ die „unreifen“ weit weniger stimulatorisch aktiv befanden als die „reifen“ MoDZ. Die Diskrepanzen könnten sich durch Verwendung des nicht standardisierbaren FCS erklären. In 2 von G. Schuler gemeinsam mit E. Kämpgen (Würzburg) und A. Enk (Mainz) [23, 26] durchgeführten Phase-I-Studien konnte durch serielles Immunomonitoring erstmals gezeigt werden, dass eine DZ-Vakzination selbst bei fortgeschrittenen Tumorpatienten (Stadium-IV-Melanom) regelmäßig tumorspezifische Immunantworten (Effektoren und/oder CTL-Präkursoren) induzieren kann (proof of principle), die nach 3 Vakzinationen (alle 14 Tage 6 Mio. DZ in die Haut) mit "rei- fen Mo-DZ“ auftraten (DZ gezüchtet aus Leukaphäresaten, beladen mit Tetanustoxoid als unspezifischem Helferepitop und MAGE- 3 als einzigem Tumorpeptid sowie bei HLA-A2.1 ${ }^{+}$-Patienten auch mit Influenzamatrix-A2.1-Kontrollpeptid). Interessanterweise kam es im Gefolge der nach den 3 Injektionen in die Haut verabreichten beiden i.-v.-Impfungen $\mathrm{zu}$ einem Absinken der im Blut nachweisbaren Immunantworten. Eine von mehreren möglichen Erklärungen wäre, dass entweder die i.-v.-Route per se oder die zu häufige Applikation zu einer Herabregulation der Immunantwort führt. Trotz eindeutiger Immunantworten in allen 8 Patienten, die mit HLA-A2.1-restringiertem-MAGE-3-Peptid-beladenen $\mathrm{DZ}$ vakziniert worden waren, zeigte leider kein Einziger ein Tumoransprechen [23]. Dies könnte dadurch bedingt sein, dass das HLA-A2.1-restringierte MAGE-3-Epitop, wie seit kurzem bekannt ist, nur unzureichend von Melanomzellen präsentiert wird. Hingegen war in 6 der 11 Stadium-IV-Melanompatienten, welche mit MAGE-3A1-Peptidbeladenen DZ geimpft worden waren, die Rückbildung einzelner Metastasen zum Zeitpunkt der Evaluation 4 Wochen nach der 5. Impfung zu beobachten, obwohl alle diese Patienten (im Gegensatz zu nur $25 \%$ in der Studie von Nestle et al. [14]) chemotherapeutisch vorbehandelt und trotzdem progressiv waren. Die überlebenden Patienten wurden nach Ende der Studie mit mehreren Peptiden (zumeist auf DZ verabreicht) weiter vakziniert. Heute, etwa 2 Jahre nach Studieneintritt, leben noch 3/11 Patienten (1 davon in erst nach 1 Jahr langsamer Metastasenrückbildung eingetretener kompletter Remission) bei einer erwarteten medianen Überlebenszeit von 4 Monaten. Auch Schadendorf et al. [20] berichteten vor kurzem über eine verlängerte Überlebenszeit der Patienten in dieser Studie sowie über eine auffällige Verlangsamung des Krankheitsprozesses auch ohne CR unter DZ-Vakzination.

In einer vor dem Abschluss stehenden Nachfolgestudie unter Verwendung zahlreicher Melanompeptide konnten bereits die effektive und regelmäßige Induktion Klasse-II-restringierter, melanomspezifischer Antworten nachgewiesen werden, einem weiteren Baustein einer effektiven Immunantwort gegen das Melanom. Eine ADO-Studie, in der die wenig effektive DTIC-Standard-Thera- pie mit der DZ-Vakzination (DZ beladen mit multiplen HLA-Klasse-I- und -IIPeptiden) im Sinn einer First-line-Therapie bei Stadium-IV-Melanompatienten verglichen wird, ist angelaufen und wird vielleicht die erste randomisierte Studie auf dem Gebiete der DZ-Therapie darstellen.

Die Nachteile der DZ-Beladung mit Peptiden (z. B. Limitation auf wenige HLA-Haplotypen) können durch (z. B. RNA-)Transfektion umgangen werden, die Resultate entsprechender Studien werden in absehbarer Zeit ebenfalls zur Verfügung stehen. Neben den definierten Tumorantigenen, die erst ein Immunomonitoring und damit eine rationale Entwicklung der Vakzinationsstrategie ermöglichen, ist auch die Erfassung des gesamten Antigenspektrums der Tumoren eine interessante Möglichkeit. Dieser Ansatz, der durch Beladung der DZ mit Tumorzellprodukten (Tumor-RNA, apoptotische und/oder nekrotische Tumorzellen) oder die Fusion von DZ mit viablen Tumorzellen möglich ist, wird von manchen trotz der Nachteile (Notwendigkeit der Verfügbarkeit von Tumorzellen, schwierige Standardisierung, Gefahr der Autoimmunität) a priori favorisiert, da so vielleicht auch tumorspezifische, durch Mutationen entstandene und besser immunogene Tumorantigene miterfasst werden können. Berichte über Therapieerfolge bei metastasierendem Nierenzellkarzinom durch Fusionate zwischen Tumorzellen und allogenen (zwecks unspezifischer T-Zell-Hilfe) DZ [10] haben jüngst bei manchen Enthusiasmus für diesen Ansatz ausgelöst. Es bleibt allerdings abzuwarten, ob diese starken Effekte reproduzierbar sind und nicht nur beim immunologisch hochsensitiven Nierenzellkarzinom auftreten. Vielleicht stellen sie auch mehr eine Folge der Zytokinfreisetzung im Gefolge der Alloreaktion als ein Resultat der Wirkung klassischer, tumorspezifische T-Zellen, die durch DZ-TumorzellHybride induziert werden sollen, dar.

Zum jetzigen Zeitpunkt erscheinen die Etablierung von Qualitätskriterien und eine Standardisierung für DZ-Vakzine und Immunomonitoring dringlich. Nur so können die zahlreichen Variablen (z. B. Art, Dosis, Intervall, Route sowie Beladung der DZ) auch in kleineren Studien aussagekräftig adressiert und die DZ-Vakzinations-Strategie rasch optimiert werden. 


\section{Gentherapie beim malignen Melanom - DNA-Vakzine, Suizidgentherapie}

Das Ziel der Stimulation einer antigenspezifischen Immunantwort gegen den Tumor wird auch mit der Applikation eines alternativen Impfstoffs, der DNAVakzine, verfolgt. Die Grundlage für die Entwicklung dieser Form der Vakzine wurde durch die Charakterisierung und Isolierung der Gene, die für die tumorrelevanten Antigene kodieren, geschaffen.

DNA-Impfstoffe enthalten alle regulatorischen und kodierenden Sequenzen, die für die Expression des betreffenden Tumorantigens in einer eukaryotischen Zelle notwendig sind. Wird die DNA-Vakzine durch eine Zelle aufgenommen und gelangt sie in den Nukleus derselben, wird dort die Expression des Antigens gestartet, es wird synthetisiert und prozessiert, sodass Peptide entstehen, die im Komplex mit HLAKlasse-I-Molekülen an der Zelloberfläche präsentiert und durch zytotoxische
T-Zellen erkannt werden können. Diese Erkennung führt aber nur dann zur Stimulation einer effizienten, CTL-vermittelten Immunantwort gegen den Tumor, wenn die T-Zelle neben dem spezifischen Signal über den HLA-PeptidKomplex zusätzlich ein unspezifisches Signal über die Wechselwirkung mit kostimulatorischen Molekülen erhält. Da nur professionelle Antigen-präsentierende Zellen (APZ) solche unspezifischen Signalmoleküle an ihrer Oberfläche präsentieren können, wird die Wirksamkeit einer DNA-Vakzine entscheidend durch die Effizienz ihres Transfers in die APZ beeinflusst [5]

Die Route der Applikation bestimmt in erster Linie, welche Zellen die DNA-Vakzine aufnehmen können. Bei intradermaler Impfstoffapplikation (z. B. per Nadelinjektion oder per ballistischem Gentransfer, d. h. Bindung der DNA an Goldpartikel, Einbringen dieser Partikel in die Hautzellen durch Gasdruck) wird dieser u. a. von den Langerhans-Zellen, den „DZ“ der Haut, aufgenommen, die damit zu potenten Stimu- latoren einer Immunantwort gegen das tumorrelevante Antigen werden können. Im Fall einer i.-m.-Impfstoffinjektion wird die DNA-Vakzine eher von Myozyten als von professionellen APZ aufgenommen, da Letztere nur in sehr geringer Zahl im Muskelgewebe anzutreffen sind. Trotzdem sind auch hier die APZ für die Induktion einer Immunantwort essenziell, wobei sie das Antigen wahrscheinlich indirekt, nach dessen Synthese und Freisetzung durch die Myozyten erhalten.

Unabhängig von der Route der Applikation konnte in zahlreichen tierexperimentellen Arbeiten die Induktion spezifischer zellulärer und humoraler Immunantworten gegen das Tumorantigen nachgewiesen werden [5]. So wurde durch die Immunisierung mit DNAImpfstoffen, die für die melanomassoziierten Differenzierungsantigene TRP-1 bzw. TRP-2 kodieren, u. a. eine Protektion gegen das Mausmelanom erzielt [24, 29]. Der Erfolg der tierexperimentellen Arbeiten führte dazu, dass zu dieser Form der Vakzination sehr schnell klini-

\section{Tabelle 3}

Laufende klinische Studien in Deutschland bei Melanom

$\begin{array}{lll}\text { Peptide Adjuvans } \quad \text { HLA-Typ } & \begin{array}{l}\text { Klinische } \\ \text { Phase }\end{array} & \begin{array}{l}\text { Klinische Stadien Studienleitung } \\ \text { nach AJCC }\end{array}\end{array}$

\section{Peptidvakzination}

1.Tyrosinase

Verschiedene

$A 1, A 2, A 24, B 44$

I/II

IV NED

2.Gn TV

$-$

3. Melan A

GM-CSF

A2

Tyrosinase,

$\mathrm{A} 2$

I/II

III/IV

sp100

4.

CpG

I/II

IIIb/IV NED

Vakzination mit autologen dendritischen Zellen

1. + Melan

$-$

A2

I/II

A-Peptid

2. +21

$+/-\mathrm{IL}-2$

$A 1, A 2.1, A 3$

III/IV

II

verschiedene

Peptide

3. +22

$-$

verschiedene

$\mathrm{A1}, \mathrm{A2}, \mathrm{A} 3, \mathrm{~A} 24, \mathrm{~B} 44$

II/III

IV

Peptide

Weitere Immunisierungsansätze

1. Fusion von autologen Tumorzellen mit allogenen dendritischen Zellen

2. Fusion von autologen Tumorzellen mit allogenen dendritischen Zellen
Alle

I/II

Alle
Priv.-Doz. S. Wagner (Essen)

Prof. A. Enk (Universitätshautklinik Mainz)

Prof.D.Schadendorf (Dermatoonkologie/Mannheim) und Priv.-Doz.Dr. C. Scheibenbogen (FU Berlin)

Prof. G. Schuler (Universitätshautklinik Erlangen)

Prof. A. Knuth/ Priv.-Doz. Dr. E. Jäger

(Nordwestkrankenhaus Frankfurt)

Prof. G. Schuler (Erlangen), Prof. Fritsch (Innsbruck)

Prof. D.Schadendorf (Dermatoonkologie/Mannheim) multizentrisch im Vergleich zu DTIC

Prof.Mackensen/Priv.-Doz. Krause (Universitätsklinik Regensburg)

Dr. Trefzer/Prof.Sterry (Charité, Hautklinik, Berlin) 
sche Studien der Phase I initiiert wurden. Im Fall des Melanoms wird die Wirksamkeit einer spezifischen DNAVakzine, die für das gp1oo-melanomassoziierte Antigen kodiert, in Bezug auf die Induktion einer Immunantwort in den USA bereits im Rahmen einer Phase-II-Studie untersucht.

Die Produktion dieser Vakzinform ist kostengünstig und einfach, da sie von Bakterien übernommen wird. Die bakterielle Vakzinproduktion hat einen weiteren Vorteil: DNA, die aus Bakterien gewonnen wird, weist im Vergleich zu eukaryotischer DNA einen geringeren Methylierungsgrad auf. Es konnte gezeigt werden, dass spezifische unmethylierte Basenabfolgen in der DNA-Sequenz (so genannte CpG-Motive) einen adjuvanten Effekt haben, indem sie bei der Aufnahme durch professionelle APZ die Sekretion proinflammatorischer Zytokine induzieren [9]. Nach den Analysen zur Funktionalität dieser Vakzinform konzentrieren sich die experimentellen Arbeiten derzeit darauf, eine Optimierung des Transfers der DNA-Impfstoffe in die Zelle durch den Einsatz spezifischer Verpackungs- oder Vektorsysteme zu erzielen. Der Einschluss der DNA in Liposomen soll zu einer erhöhten Stabilität der Vakzine nach Applikation sowie zu einer verbesserten Aufnahme durch die Zellen beitragen. Auch die Wirksamkeit attenuierter Bakterien als Vektoren für einen verbesserten Vakzintransfer wird in experimentellen Arbeiten untersucht. Diese Bakterien (z. B. Salmonellen, Listerien) sind in der Lage, Zellen zu infizieren und dort die DNA freizusetzen, sodass ein Eintritt der Vakzine in den Nukleus der Zelle erleichtert wird. Zusammenfassend kann gesagt werden, dass sich die Strategie der DNA-Immunisierung zu einem bedeutenden immuntherapeutischen Werkzeug zur Behandlung von Krebs entwickeln könnte und dies besonders im Fall des Melanoms, da eine beträchtliche Zahl an Antigen-kodierenden Genen bekannt ist, welche die Grundlage für die Entwicklung dieses Impfstoffs darstellen.

Die beschriebene Form der ,genetischen" Immuntherapie ist jedoch nur eine Variante der Gentherapie zur Behandlung von Tumoren. Das ständig zunehmende Wissen um die molekulare Basis des Tumors führte zur Entwicklung neuer Strategien, in denen genetisches Material zur Bekämpfung des Tumors eingesetzt wird. Es werden u. a. Methoden zur genetischen Korrektur des malignen Phänotyps einer Tumorzelle entwickelt (so genannte Mutationskompensation) sowie Strategien zur Inhibition der tumorinduzierten Angiogenese erarbeitet.

Das Prinzip der Suizidgentherapie verfolgt das Ziel, den Selbstmord des Tumors durch die Expression toxischer Gene in den Tumorzellen einzuleiten. Das Toxingen wird in Form einer therapeutischen DNA appliziert, die von der Tumorzelle aufgenommen und nach Eintritt in den Nukleus exprimiert wird. Dabei kodiert das Toxingen in der Regel für ein spezifisches Enzym, das die Umwandlung einer nichttoxischen Vorstufe eines Medikaments in eine toxische Substanz katalysiert. Als Beispiel sei die Herpes-simplex-Virus-Thymidinkinase (HSV-TK) genannt, die das Medikament Ganciclovir (GCV) in GCV-Monophosphat umwandelt, das schließlich in eine für die Zelle toxische Triphosphatform überführt wird. Zahlreiche In-vitro- und Invivo-Analysen belegen, dass es im Fall der HSV-TK zu einem so genannten „Bystander"-Effekt kommt, der dazu führt, dass auch benachbarte Zellen, die das Toxingen selbst nicht exprimieren, abgetötet werden. Demzufolge ist es nicht notwendig, dass wirklich jede Tumorzelle die therapeutische DNA aufnimmt. Aufgrund des Wirkmechanismus dieser Therapie ist es jedoch essenziell, die Expression des Toxingens auf die Zellen des Tumors zu begrenzen, um eine Schädigung gesunden Gewebes zu begrenzen. Solch eine zelltypspezifische Toxinsynthese kann dadurch erreicht werden, dass bereits die Phase der Initiation der Genexpression durch spezifische regulatorische DNA-Sequenzen kontrolliert wird. Um eine gewebespezifische Expression in Melanomzellen zu erzielen, wurde in vielen experimentellen Studien der Tyrosinasepromotor zur Expression des Toxingens eingesetzt [4]. Durch die intratumorale Injektion solch therapeutischer DNA konnten dann im murinen Melanommodell beeindruckende Tumorregressionen induziert werden [17]. Allerdings wird die Wirksamkeit einer solchen gentherapeutischen DNA aufgrund bislang nicht vorhandener effizienter Vektorsysteme, die eine systemische Applikation nicht erlauben, stark eingeschränkt. Letztlich ist dies ein Problem vieler Strategien der DNAGentherapie, sodass intensive Forschungsarbeit auf dem Gebiet der Herstellung geeigneter Vektoren geleistet wird. Sollten diese Vektoren zur Verfügung stehen, würde dies die Translation präklinischer Studien in die klinische Anwendung beschleunigen.

\section{Perspektiven}

Experimentelle Therapieansätze, basierend auf tumorimmunologischen Prinzipien, sind derzeit ein Hot-spot der (prä-) klinischen Forschung. Wenngleich es in einzelnen Tiermodellen gelingt, Tumorprogression oder -ausbreitung mit Immunisierungsstrategien zu bekämpfen, ist dies noch ein langer Weg bis zu einer etablierten Tumortherapie in Klinik und Praxis. Derzeit sind wichtige Fragen, wie ein geeignetes Immunomonitoring während und nach der Impfung durchzuführen ist, nicht geklärt. Oft ist es schwer, signifikante T-Zell-Antworten im peripheren Blut nachzuweisen, und der Nachweis bzw. das Fehlen solcher T-Zell-Antworten korreliert nicht mit dem (seltenen) klinischen Ansprechen. Insofern ist anzunehmen, dass der Immunisierungseffekt, der derzeit erzielt wird - im Gegensatz zu herkömmlichen Schutzimpfungen z. B. gegen Viren - noch viel zu schwach ist. Erst wenn es reproduzierbar gelingt, starke Immunantworten im $\mathrm{Tu}$ morpatienten $\mathrm{zu}$ generieren, wird es möglich sein, eine weitere Problemzone $\mathrm{zu}$ bearbeiten. Diese ist der so genannte „immune escape“ d. h. die Fähigkeit von Tumorzellen, einzelne HLA-Moleküle oder Tumorantigene herunterzuregulieren bzw. abzuschalten. Sowohl durch das zunehmend verbesserte tumorimmunologische Verständnis als auch durch das Wachsen von identifizierten Tumorantigenen bestehen hier aber sicherlich Möglichkeiten, effektive Ansätze zu entwickeln, um dieses Problem anzugehen. Eine Möglichkeit ist die Verwendung von multiplen Peptiden.

\section{Fazit für die Praxis}

Zusammenfassend lässt sich feststellen, dass die Tumorimmunologie in der letzten Dekade mit der Identifikation von Molekülen im Tumor, die vom menschlichen Immunsystem erkannt werden, einer deutlichen Aufschwung genommen hat. Parallel haben die wachsenden Erkenntnisse über die Arbeitsweise des Immunsystems Möglichkeiten eröffnet, Strategien zu erarbeiten, um auch Tumoren zu bekämpfen. Al- 
lerdings steht die Umsetzung in die tägliche klinische Anwendung noch ganz am Anfang. Derzeit aktuelle klinische Studien sind in Tabelle 3 aufgeführt.

Weiterhin bleibt zu berücksichtigen, dass die Tumorentstehung und das Tumorwachstum außerordentlich komplex und damit auch äußerst schwierig therapeutisch anzugehen sind. Darüber hinaus sind Tumorzellen, "lebende Organismen", die teilweise sehr gut in der Lage sind, eigene Abwehrstrategien gegen das Immunsystem zu entwickeln. Diese Situation ist in der weit fortgeschrittenen Krankheitssituation besonders ausgeprägt. Idealerweise sollten daher Patienten möglichst frühzeitig, z. B. nach Entfernung des Primärtumors, vorbeugend im Sinn einer „Schutzimpfung" behandelt werden. Erstmals erscheint es jetzt realistisch, dass dieser Traum tatsächlich Realität werden könnte. Allerdings wird es noch Jahre dauern, bis klar sein wird, welche Patientengruppe wie am meisten profitiert.

\section{Literatur}

1. Bell D, Young JW, Banchereau J (1999) Dendritic cells. Adv Immunol 72:255-324

2. Cormier JN, Salgaller ML, Prevette T, Barracchini KC, Rivoltini L, Restifo NP, Rosenberg SA, Marincola FM (1996) Enhancement of cellular immunity in melanoma patients immunized with a peptide from MART-1/melan A. Cancer J Sci Am 3:37-44

3. Dhodapkar MV, Krasovsky J, Steinman RM, Bhardwaj N (2000) Mature dendritic cells boost functionally superior CD8+ T-cells in humans without foreign helper epitopes. $\mathrm{J}$ Clin Invest 105:R9-R14

4. Diaz RM, Eisen T, Hart IR, Vile RG (1998) Exchange of viral promoter/ enhancer elements with heterologous regulatory sequences generates targeted hybrid long terminal repeat vectors for gene therapy of melanoma. JVirol 72:789-795

5. Donnelly JJ, Ulmer JB, Shiver JW, Liu MA (1997) DNA vaccines. Annu Rev Immunol 15:617-648

6. Inaba K (1990) Dentritic cells pulsed with protein antigens in vitro can prime antigenspecific, MHC-restricted T cells in situ. J Exp Med 172:631-640

7. Jaeger $E$, Ringhoffer $M$, Dienes HP, Arand M, Karbach J, Jaeger D, Ilsemann C, Hagedorn M, Oesch F, Knuth A (1996) GM-CSF enhances immune responses to melanoma-associated peptides in vivo. Int J Cancer 67:54-62

8. Keilholz U, Scheibenbogen C, Hofmann, U, Servetopulou F, Schmittel A, Siehl J, Foerster MH, Thiel D, Schadendorf D (2000) Therapeutic versus adjuvant peptide vaccination: comparison of two phase I/II trials in melanoma aptients. Proceedings ASCO Abstract 2191
9. Krieg AM (1999) Direct immunologic activities of CPG DNA and implications for gene therapy. J Gene Med 1:56-63

10. Kugler A, Stuhler $G$, Walden $P$, Zoller $G$, Zobywalski A, Brossart P, Trefzer U, Ullrich S, Muller CA, Becker V, Gross AJ, Hemmerlein B, Kanz L, Muller GA, Ringert RH (2000) Regression of human metastatic renal cell carcinoma after vaccination with tumor cell-dendritic cell hybrids. Nat Med 6:332-336

11. Lewis JJ, Janetzki S, Schaed S, Panageas C, Wang S, Williams L, Myers M, Butterworth L, Livingston P, Chapman P, Houghton A (2000) Evaluation of $C D 8+T$ cell frequencies by the ELISPOT assay in healthy individuals and patients with metastatic melanoma immunized with tyrosinase peptide. Int J Cancer 87:391-398

12. Mackensen A, Herbst B, Chen JL, Kohler G, Noppen C, Herr W, Spagnoli GC, Cerundolo V, Lindemann A (2000) Phase I study in melanoma patients of a vaccine with peptide-pulsed dendritic cells generated in vitro from CD34(+) hematopoietic progenitor cells. Int J Cancer 86:385-392

13. Marchand M, Van Baren N, Weynants $P$, Brichard V, Dreno B, Tessier MH, Rankin E, Parmiani G, Arienti F, Humblet Y, Bourlond A, Vanwijck R, Lienard D, Beauduin M, Dietrich PY, Russo V, Kerger J, Masucci G, Jager E, De Greve J, Atzpodien J, Brasseur F, Coulie PG, Van der Bruggen P, Boon T (1999) Tumor regressions observed in patients with metastatic melanoma treated with an antigenic peptide encoded by gene MAGE-3 and presented by HLA-A1. Int J Cancer 80:219-230

14. Nestle FO, Alijagic S, Gilliet M, Sun Y, Grabbe S, Dummer R, Burg G, Schadendorf D (1998) Vaccination of melanoma patients with peptide- or tumor lysate-pulsed dendritic cells. Nat Med 4:328-332

15. Oettgen HF, Old LJ (1991) The history of cancer immunotherapy. In: DeVita VT, Helman S, Rosenberg SA (eds) Biologic therapy of cancer, principles and practice. Lippincott, Philadelphia, pp 87-97

16. Pardoll DM, Beckerleg AM (1995) Exposing the immunology of naked DNA vaccines. Immunity 3:165-169

17. Ramesh R, Munshi A, Marrrogi AJ, Freeman SM (1999) Enhancement of tumor killing using a combination of tumor immunization and HSV-tk suicide gene therapy. Int J Cancer 80:380-386

18. Rosenberg $S A$, Yang JC, Schwartzentruber DJ, Hwu P, Marincola FM, Topalian SL, Restifo NP, Dudley ME, Schwarz SL, Spiess PJ, Wunderlich JR, Parkhurst MR, Kawakami Y, Seipp CA, Einhorn JH, White DE (1998) Immunologic and therapeutic evaluation of asynthetic peptide vaccine for the treatment of patients with metastatic melanoma. Nat Med 4:321-327

19. Schadendorf D, Nestle FO (2000) Autologous dendritic cells for treatment of advanced cancer - an update - .

Recent Results Cancer Res 158:236-248
20. Schadendorf D, Grabbe S, Nestle FO (1997) Vaccination with dendritic cells - a specific immunomodulatory approach for the treatment of malignant melanoma. In: Burg G, Dummer R (eds) Strategies for immunointervention in dermatology. Springer, Berlin Heidelberg New York, pp 399-409

21. Scheibenbogen C, Schmittel A, Keilholz U, Allgäuer T, Hofmann U, Max R, Thiel E, Schadendorf D (2000) Phase II trial of vaccination with tyrosinase peptides and GM-CSF in melanoma.J Immunother 23:275-281

22. Schuler G, Steinman RM (1996) Dendritic cells as adjuvants for immune-mediated resistance to tumors.J Exp Med 186:1183-1187

23. Schuler-Thurner B, Dieckmann D, Keikavoussi P, Bender A, Maczek C, Jonuleit H, Röder C, Händle I, Leisgang W, Dunbar R, Cerundolo V, Driesch P von den, Knop J, Bröcker EB, Enk A, Kämpgen E, Schuler G (2000) Mage-3 and influenza-matrix peptide-specific cytotoxic $T$ cells are inducible in terminal stage HLA-A2. $1+$ melanoma patients by mature monocytederived dendritic cells. I Immunol 165:3492-3496

24. Steitz J, Bruck J, Steinbrink K, Enk A, Knop J, Tuting T (2000) Genetic immunization of mice with human tyrosinase-related protein 2: implications for the immunotherapy of melanoma. Int J Cancer 86:89-94

25. Sun Y, Paschen A, Schadendorf D (1999) Cellbased vaccination against melanoma - background, preliminary results and perspectives. J Mol Med 73:593-608

26. Thurner B, Haendle I, Röder C, Dieckmann D, Keikavoussi P, Jonuleit H, Bender A, Maczek C, Schreiner D, Driesch P von den, Bröcker EB, Steinman RM, Enk A, Kämpgen E, Schuler G (1999) Vaccination with mage-3A1 peptidepulsed mature, monocyte-derived dendritic cells expands specific cytotoxic T cells and induces regression of some metastases in advanced stage IV melanoma.J Exp Med 190:1669-1678

27. Wang RF (1999) Human tumor antigens: implications for cancer vaccine development. J Mol Med 77:640-655

28. Wang F, Bade E, Kuniyoshi C, Spears L, Jeffrey G, Marty V, Groshen S, Weber J (1999) Phase I trial of a MART-1 peptide vaccine with incomplete Freund's adjuvant for resected high-risk melanoma. Clin Cancer Res 5:2756-2765

29. Weber LW, Bowne WB, Wolchok JD, Srinivasan R, Qin J, Moroi Y, Clynes R, Song P, Lewis JJ, Houghton AN (1998) Tumor immunity and autoimmunity induced by immunization with homologous DNA.J Clin Invest 102:1258-1264

30. Weber JS, Hua FL, Spears L, Marty V, Kuniyoshi C, Celis E (1999) A phase I trial of an HLA-A1restricted MAGE-3 epitope peptide with IFA in patients with resected high-risk melanoma. J Immunother 5:431-440

31. Weber J, Jeffrey G, Marty V, Bade E, Lau R, Wolf S, Lee P,Wang $F$ (2000) Phase II trial of gp100/ tyrosinase peptides/IFA with and without IL-12 for resected stages III/IV melanoma. Proceedings ASCO, Abstract 1786 